\title{
Treatment Duration, Healthcare Resource Utilization, and Costs Among Chemotherapy-Naïve Patients with Metastatic Castration-Resistant Prostate Cancer Treated with Enzalutamide or Abiraterone Acetate: A Retrospective Claims Analysis
}

\author{
Neil M. Schultz · Scott C. Flanders · Samuel Wilson • Bruce A. Brown · \\ Yan Song · Hongbo Yang · Stanislav Lechpammer · Vahan Kassabian
}

Received: June 26, 2018 / Published online: September 6, 2018

(C) The Author(s) 2018

\section{ABSTRACT}

Introduction: Enzalutamide and abiraterone acetate (plus prednisone) are new hormonal treatments for metastatic castration-resistant prostate cancer (mCRPC). This study compared treatment duration, healthcare resource utilization (HRU), and treatment costs for chemotherapy-naïve mCRPC patients treated with enzalutamide or abiraterone acetate in the USA.

Enhanced digital content To view enhanced digital content for this article go to https://doi.org/10.6084/ m9.figshare.6949874.

Electronic supplementary material The online version of this article (https://doi.org/10.1007/s12325018-0774-1) contains supplementary material, which is available to authorized users.

N. M. Schultz $(\bowtie) \cdot$ S. C. Flanders · S. Wilson .

B. A. Brown

Astellas Pharma Inc., 1 Astellas Way, Northbrook, IL 60062, USA

e-mail: neil.schultz@astellas.com

Y. Song · H. Yang

Analysis Group, Inc., 111 Huntington Avenue, 14th

Floor, Boston, MA 02199, USA

S. Lechpammer

Pfizer Inc., 525 Market Street, 36th Floor,

San Francisco, CA 94105, USA

V. Kassabian

Georgia Urology, Fulton County, 5730 Glenridge

Drive, Suite 200, Atlanta, GA 30328, USA
Methods: Chemotherapy-naïve mCRPC patients initiating treatment with enzalutamide or abiraterone acetate were identified from administrative claims. Continuous enrollment $\geq 6$ months before and $\geq 3$ months after the index date (initiation date of enzalutamide or abiraterone acetate) was required. Treatment duration, all-cause and prostate cancer-related HRU, and costs were estimated during the postindex period. Multivariable analyses compared HRU and costs between cohorts, adjusting for baseline characteristics.

Results: Overall, 920 chemotherapy-naïve patients initiated enzalutamide and 2310 initiated abiraterone acetate (median follow-up, 10.7 and 13.5 months, respectively). More enzalutamide-treated patients had corticosteroid-sensitive comorbidities at baseline. Treatment duration was longer with enzalutamide versus abiraterone acetate (median, 10.7 vs. 8.8 months; $P=0.008$ ). Enzalutamide was associated with fewer all-cause inpatient admissions [adjusted incidence rate ratio (95\% confidence interval) $0.87(0.76,0.99)]$, days of hospitalization $[0.84(0.70,1.02)]$, and outpatient visits $[0.94(0.90,0.98)]$, and fewer prostate cancer-related outpatient visits $[0.92$ (0.87, 0.96)] compared with abiraterone acetate. Enzalutamide was also associated with lower prostate cancer-related inpatient and emergency department costs [adjusted differences, $\$ 122 \quad(P=0.024)$ and $\$ 28 \quad(P=0.009)$, respectively]. 
Conclusion: Chemotherapy-naïve mCRPC patients treated with enzalutamide versus abiraterone acetate had longer treatment duration and incurred lower HRU and prostate cancerrelated inpatient and emergency department costs.

Funding: Astellas Pharma Inc.

Keywords: Abiraterone acetate; Chemotherapy naïve; Claims analysis; Enzalutamide; Healthcare costs; Hormonal therapy; Metastatic castration-resistant prostate cancer; Oncology; Retrospective study; Treatment patterns

\section{INTRODUCTION}

Prostate cancer is the most commonly diagnosed cancer among men worldwide and the second leading cause of cancer-related deaths $[1,2]$. Castration-resistant prostate cancer (CRPC) is an advanced form of prostate cancer characterized by disease progression following surgical castration or androgen-deprivation therapy $[3,4]$. Approximately $10-20 \%$ of all prostate cancer is CRPC, with over $84 \%$ demonstrating radiographic findings of metastatic CRPC (MCRPC) [4]. MCRPC is associated with a poor prognosis and reduced survival compared with CRPC, and no curative treatments are available $[4,5]$.

Until 2010, treatments of mCRPC were primarily limited to taxane chemotherapy (docetaxel) or oral non-steroidal anti-androgen therapy plus luteinizing hormone-releasing hormone analogs [6]. Recently, there have been many advances in treatment options, including novel taxane chemotherapy (e.g., cabazitaxel), immunotherapy (e.g., sipuleucel-T), radiotherapy (e.g., radium-223), and novel hormonal therapy (e.g., enzalutamide and abiraterone acetate) [7]. These new treatments have greatly extended the median overall survival of patients with mCRPC (ranging from approximately 918 months in 2010 to $16-35$ months in 2016), depending on tumor burden $[4,8,9]$.

The US Food and Drug Administration approved abiraterone acetate and enzalutamide for mCRPC patients with prior chemotherapy in
April 2011 and August 2012, respectively, and for chemotherapy-naïve patients in December 2012 and September 2014, respectively [10]. Although both are androgen-directed hormonal agents, the two drugs act via different mechanisms; abiraterone acetate inhibits androgen synthesis while enzalutamide blocks the androgen-receptor signaling pathway [11]. In clinical trials, both treatments have prolonged overall survival in chemotherapy-naïve patients with mCRPC compared with placebo (enzalutamide, 35.3 vs. 31.3 months with placebo; abiraterone acetate plus prednisone, 34.7 vs. 30.3 months with placebo plus prednisone) $[12,13]$. Enzalutamide and abiraterone acetate have also received the highest recommendation (category 1) in the National Comprehensive Cancer Network guidelines for the treatment of mCRPC [14].

Although the results of clinical trials demonstrate the efficacy of enzalutamide and abiraterone acetate for mCRPC under controlled conditions, it is as important to evaluate these treatments in real-world clinical practice to assess their impact on patient outcomes. While enzalutamide and abiraterone acetate have been used with patients with mCRPC for several years, there is limited published real-world evidence of their associated treatment outcomes. A few studies have compared pharmacy costs $[15,16]$ or evaluated treatment duration and dose reduction for the overall population of prostate cancer patients receiving enzalutamide or abiraterone acetate [7, 17]. However, many mCRPC patients do not receive chemotherapy as the initial treatment [16], and previous research has suggested greater benefits of enzalutamide and abiraterone acetate in chemotherapy-naïve patients [18, 19]. These benefits include delay of the initiation of cytotoxic chemotherapy, which could be of clinical importance for patients with pre-existing medical conditions and who may have lower tolerability for the toxicity profile of chemotherapy $[20,21]$. Thus, it is particularly important to assess treatment outcomes in the chemotherapy-naïve patient population with mCRPC.

To date, no studies have examined realworld outcomes, including treatment duration, healthcare resource utilization (HRU), and 
associated medical costs of chemotherapy-naïve mCRPC patients treated with enzalutamide or abiraterone acetate. Additionally, no published studies assessed real-world outcomes among specific comorbid disease subpopulations of patients with mCRPC, even though treatment response may depend on patients' pre-existing comorbidities, and this topic has not been explored in the literature.

Therefore, to address these gaps in the existing literature and contribute additional evidence to the current body of research in mCRPC, the current study evaluates the realworld treatment duration, HRU, and medical costs for patients with mCRPC treated with enzalutamide and abiraterone acetate using administrative claims data. In addition, this is the first study to assess the real-world outcomes in subgroups of chemotherapy-naïve patients with mCRPC, namely those with diabetes, cardiovascular diseases, and corticosteroid-sensitive comorbidities.

\section{METHODS}

\section{Data Source}

This study used the Truven Health MarketScan ${ }^{\circledR}$ Commercial Claims and Encounters and Medicare Supplemental Databases (2012-2015). The commercial database contains the combined claims of employees and dependents, totaling approximately 147 employers with 16 health plans, while the Medicare supplemental database covers Medicare-eligible retirees with employer-sponsored Medicare supplemental plans. The databases include enrollment history and claims for medical (provider and institutional) and pharmacy services. As this was a retrospective analysis of anonymized data, no institutional board review was required.

\section{Study Design and Patient Populations}

The date of the first claim of enzalutamide or abiraterone acetate was defined as the index date. Enzalutamide or abiraterone acetate received on the index date was defined as the index treatment. The baseline period was defined as the 6-month period before the index date. The study period spanned from the index date until the end of data availability (the end of continuous enrollment in the insurance plan), with a minimum of 3 months of followup required.

\section{Inclusion Criteria}

The patient selection diagram is shown in Fig. 1. Male patients were included in this study if they had at least one pharmacy claim for enzalutamide or abiraterone acetate on or after 1 September 2012 (the earliest time both treatments were commercially available in the USA). Eligible patients were at least 18 years old at the index date and were required to have a unique index treatment (i.e., they did not initiate enzalutamide/abiraterone acetate combination on the same day). Additionally, patients were required to have at least one prostate cancer diagnosis [International Classification of Disease, Ninth Revision, Clinical Modification (ICD-9-CM) code 185 or Tenth Revision code C61] during the 6 months before the index date and at least two prostate cancer diagnoses on distinct dates in the entire claims history; continuous enrollment for at least 6 months before and at least 3 months after the index date; and no claims for cytotoxic chemotherapy in the entire claims history before the index date. Continuous enrollment was defined as having no gap between periods of enrollment.

\section{Patient Subgroups}

The following patient subgroups were defined according to the baseline comorbidity profile: patients with diabetes (including both type 1 and type 2 diabetes mellitus); patients with cardiovascular diseases (including hypertension, ischemic heart disease, cerebrovascular disease, peripheral vascular disease, and congestive heart failure); and patients with corticosteroid-sensitive comorbidities [22] (including diabetes, cataracts, glaucoma, hypertension, hyperlipidemia, ischemic heart disease, cerebrovascular disease, peripheral vascular disease, congestive heart failure, osteoporosis, anxiety, depression, insomnia, 


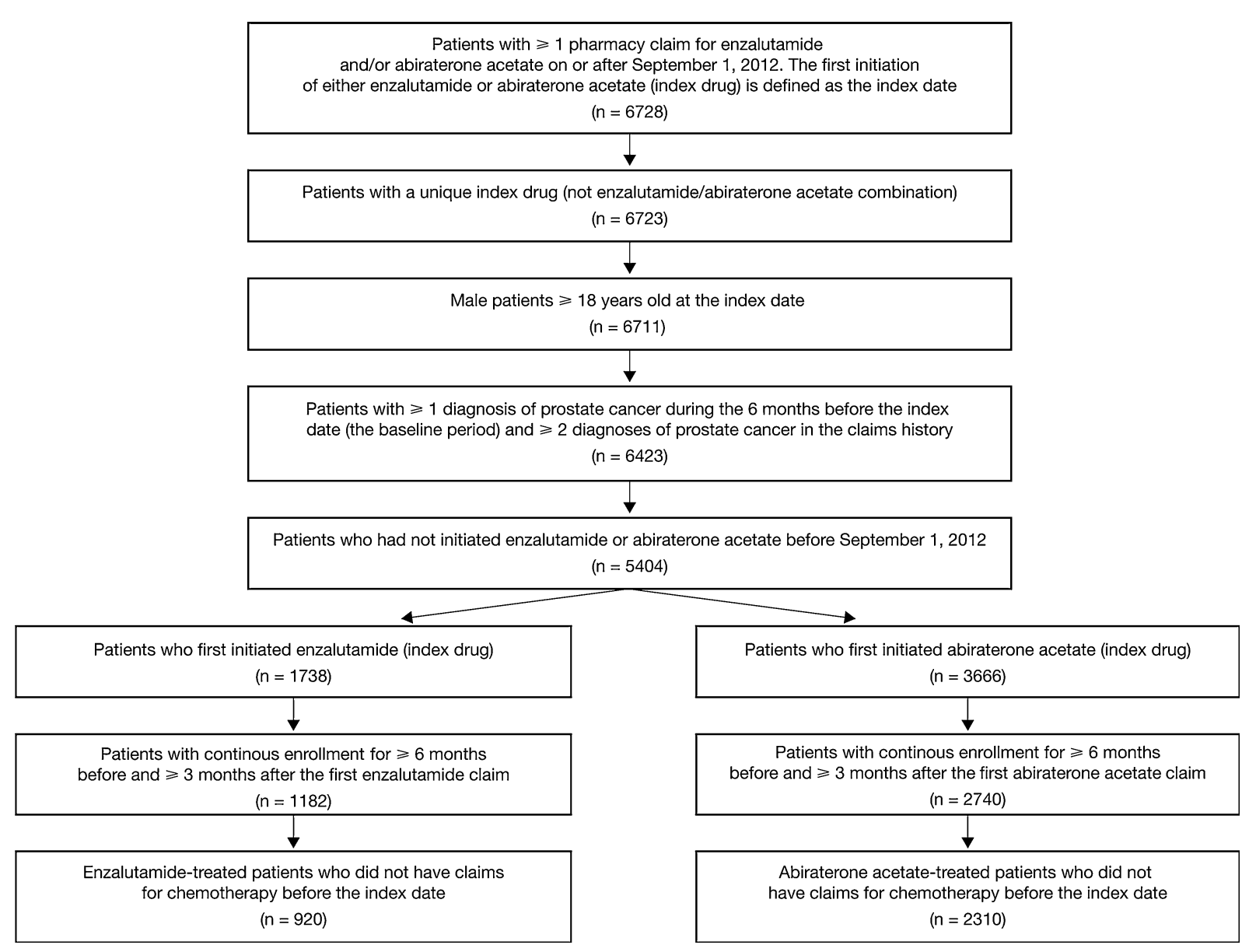

Fig. 1 Sample selection flowchart. September 1, 2012, was the earliest time at which enzalutamide and abiraterone acetate were both commercially available in the US.

Continuous enrollment was defined as having no gap between periods of enrollment

schizophrenia, bipolar disorder, and gastrointestinal ulcer and perforation).

\section{Study Covariates and Outcomes}

\section{Baseline Patient Characteristics}

The following baseline patient characteristics were assessed during the baseline period: age at index date; year of index date; region of residence; health insurance type; prostate cancerrelated comorbidities; Charlson Comorbidity Index (CCI) [23] and chronic comorbidities; and treatments received for prostate cancer.

\section{Treatment Duration}

Treatment duration was calculated from the initiation date of the index treatment to

discontinuation. Discontinuation was defined as a gap of at least 45 days between the end of the supply of one fill for the index treatment and the date of the next fill. Sensitivity analyses were also conducted, with discontinuation defined as a gap of at least 30 or 60 days. Patients who did not discontinue the index treatment were censored at the end of data availability.

\section{HRU and Costs}

All-cause HRU, defined as the total counts of healthcare encounters regardless of underlying medical reasons, was quantified during the study period and included the following categories: inpatient admissions and total days of hospitalization; emergency department visits; 
and outpatient visits. All-cause medical service costs and pharmacy costs were assessed during the study period. Specific cost categories, including inpatient, outpatient, emergency department, and index-drug pharmacy costs, were assessed. The costs were adjusted to 2017 US dollars according to the medical care component of the Consumer Price Index. The cost analysis was restricted to patients who were not on capitated insurance plans to ensure that service costs were accurately represented.

The same outcomes as all-cause HRU and costs were assessed for prostate cancer-related HRU and costs, defined as encounters and medical claims with a primary or secondary diagnosis of prostate cancer. Prostate cancerrelated pharmacy costs were defined as any claims for prescription drugs related to prostate cancer.

\section{Statistical Analyses}

For comparisons of baseline characteristics, continuous variables were summarized using means and standard deviations (SDs), with categorical variables summarized using counts and proportions. Treatment duration was estimated using Kaplan-Meier analyses and compared using unstratified log-rank tests between the enzalutamide- and abiraterone acetate-treated patients. Median time on treatment and proportions of patients who discontinued the index treatment at 6,12 , and 24 months after initiation were reported.

Monthly rates of HRU were estimated by dividing the total number of events by the total person-months from the index date through the end of the study period for each cohort. The monthly rates allowed for comparisons between groups comprising patients with different follow-up times using all available data. In addition, the presence of at least one HRU event was evaluated within the first 3-month period after the index date. Incidence rate ratios (IRRs) for HRU outcomes and 95\% confidence intervals (CIs) were estimated using generalized linear models with a Poisson distribution, and odds ratios (ORs) were estimated using logistic regression models.
Monthly costs were calculated for each patient by dividing the total costs accrued during the study period by the length of follow-up from the index date. All-cause and prostate cancer-related monthly costs were summarized using means and SDs. The differences in costs were then estimated using generalized linear models with Tweedie distribution (a compound Poisson-gamma distribution) [24-26].

Both unadjusted and adjusted comparisons of HRU and costs were conducted among the overall population and among patient subgroups as defined previously. Adjusted comparisons were controlled for the following baseline covariates selected based on clinical relevance: patient age at the index date; $\mathrm{CCI}$; year of the index date; number of all-cause and prostate cancer-related inpatient and outpatient visits during the baseline period; surgical or chemical castration received during the baseline period; and the use of any anti-androgen or androgen synthesis inhibitor during the baseline period.

All statistical analyses were performed using SAS $^{\circledR}$ Studio 3.5 (SAS Institute Inc., Cary, NC, USA). $P<0.05$ was considered statistically significant.

\section{RESULTS}

A total of 6728 male adult patients were identified with at least one diagnosis of prostate cancer during the baseline period and at least one pharmacy claim for enzalutamide or abiraterone acetate on or after September 1, 2012 (Fig. 1). Of these, 920 patients initiating enzalutamide and 2310 patients initiating abiraterone acetate did not have a claim for chemotherapy before the index date and met all other criteria for inclusion in the analysis. The median follow-up time was 10.7 months for patients in the enzalutamide cohort and 13.5 months for patients in the abiraterone acetate cohort.

\section{Baseline Characteristics}

Patients' characteristics at baseline are listed in Table 1. On average, enzalutamide-treated 
Table 1 Patient demographics and baseline characteristics

\begin{tabular}{|c|c|c|}
\hline Patient characteristics & Enzalutamide $(n=920)$ & Abiraterone acetate $(n=2310)$ \\
\hline \multicolumn{3}{|l|}{ Demographics } \\
\hline Age, mean $\pm S D$ & $74.5 \pm 10.7$ & $73.5 \pm 10.6$ \\
\hline \multicolumn{3}{|l|}{ Region, $n(\%)$} \\
\hline Northeast & $224(24.3)$ & $559(24.2)$ \\
\hline North Central & $282(30.7)$ & $638(27.6)$ \\
\hline South & $290(31.5)$ & $685(29.7)$ \\
\hline West & $121(13.2)$ & $418(18.1)$ \\
\hline Unknown & $3(0.3)$ & $10(0.4)$ \\
\hline \multicolumn{3}{|l|}{ Year of index date, $n(\%)$} \\
\hline 2012 & $48(5.2)$ & $239(10.3)$ \\
\hline 2013 & $159(17.3)$ & $1096(47.4)$ \\
\hline 2014 & $275(29.9)$ & $621(26.9)$ \\
\hline 2015 & $438(47.6)$ & $354(15.3)$ \\
\hline Medicare supplemental coverage, $n(\%)$ & $725(78.8)$ & $1732(75.0)$ \\
\hline \multicolumn{3}{|l|}{ Health insurance type, $n$ (\%) } \\
\hline Comprehensive & $348(37.8)$ & $806(34.9)$ \\
\hline $\mathrm{PPO}$ & $396(43.0)$ & $1013(43.9)$ \\
\hline HMO and other capitated plans & $73(7.9)$ & $295(12.8)$ \\
\hline Other & $103(11.2)$ & $196(8.5)$ \\
\hline \multicolumn{3}{|l|}{ Comorbidities } \\
\hline $\mathrm{CCI},{ }^{\mathrm{a}}$ mean $\pm \mathrm{SD}$ & $2.7 \pm 1.2$ & $2.6 \pm 1.1$ \\
\hline \multicolumn{3}{|l|}{ Prostate cancer-related comorbidities, $n$ (\%) } \\
\hline Bone metastases & $564(61.3)$ & $1481(64.1)$ \\
\hline Hypertension & $526(57.2)$ & $1195(51.7)$ \\
\hline Urinary tract infection & $95(10.3)$ & $245(10.6)$ \\
\hline Glaucoma & $81(8.8)$ & $232(10.0)$ \\
\hline Depression & $51(5.5)$ & $108(4.7)$ \\
\hline Impotence & $44(4.8)$ & $111(4.8)$ \\
\hline \multicolumn{3}{|l|}{ Other comorbidities, $n$ (\%) } \\
\hline Diabetes & $253(27.5)$ & $533(23.1)$ \\
\hline Chronic pulmonary disease & $124(13.5)$ & $275(11.9)$ \\
\hline Malignancies (excluding prostate cancer) & $131(14.2)$ & $368(15.9)$ \\
\hline Renal disease & $108(11.7)$ & $272(11.8)$ \\
\hline
\end{tabular}


Table 1 continued

\begin{tabular}{|c|c|c|}
\hline Patient characteristics & Enzalutamide $(n=920)$ & Abiraterone acetate $(n=2310)$ \\
\hline Peripheral vascular disease & $107(11.6)$ & $245(10.6)$ \\
\hline Congestive heart failure & $91(9.9)$ & $182(7.9)$ \\
\hline Cerebrovascular disease & $71(7.7)$ & $183(7.9)$ \\
\hline Liver disease & $44(4.8)$ & $160(6.9)$ \\
\hline Myocardial infarction & $29(3.2)$ & $61(2.6)$ \\
\hline \multicolumn{3}{|c|}{ Treatments received during baseline period, $n(\%)$} \\
\hline \multicolumn{3}{|l|}{ Pharmaceutical treatments } \\
\hline \multicolumn{3}{|l|}{ LHRH agonists/antagonists ${ }^{\mathrm{b}}$} \\
\hline During baseline period & $675(73.4)$ & $1645(71.2)$ \\
\hline Any time before index date & $770(83.7)$ & $1879(81.3)$ \\
\hline Anti-androgen ${ }^{c}$ & $450(48.9)$ & $1334(57.7)$ \\
\hline Opioid analgesics & $394(42.8)$ & $984(42.6)$ \\
\hline Osteoclast inhibitors $^{\mathrm{d}}$ & $338(36.7)$ & $823(35.6)$ \\
\hline Corticosteroids & $184(20.0)$ & $1054(45.6)$ \\
\hline Sipuleucel-T & $76(8.3)$ & $147(6.4)$ \\
\hline Radiopharmaceuticals $^{\mathrm{e}}$ & $5(0.5)$ & $3(0.1)$ \\
\hline \multicolumn{3}{|l|}{ Procedures } \\
\hline Surgical castration ${ }^{\mathrm{f}}$ & $15(1.6)$ & $37(1.6)$ \\
\hline Radiation $^{\mathrm{g}}$ & $13(1.4)$ & $9(0.4)$ \\
\hline
\end{tabular}

CCI Charlson Comorbidity Index, HMO health maintenance organization, LHRH luteinizing hormone-releasing hormone, $P P O$ preferred provider organization, $S D$ standard deviation

a The CCI has been modified to exclude prostate cancer and metastatic disease

b LHRH agonists/antagonists included leuprolide, goserelin, triptorelin, histrelin, degarelix, and diethylstilbestrol

c Anti-androgens included bicalutamide, nilutamide, and flutamide

d Osteoclast inhibitors included denosumab and zoledronic acid

e Radiopharmaceuticals included radium-223 and samarium-153

f Surgical castration included both unilateral and bilateral orchiectomy

g Radiation included external beam radiation therapy, stereotactic radiation therapy, and hemibody irradiation

patients were 1 year older than patients treated with abiraterone acetate [74.5 (SD, 10.7) vs. 73.5 (10.6) years, respectively]. With respect to comorbidities, patients in both cohorts had similar mean CCI scores [enzalutamide, 2.7 (SD, 1.2); abiraterone acetate, 2.6 (1.1)]. However, larger proportions of enzalutamide- versus abiraterone acetate-treated patients had certain corticosteroid-sensitive comorbidities, including hypertension $(57.2 \%$ vs. $51.7 \%$, respectively) and diabetes $(27.5 \%$ vs. $23.1 \%$, respectively). Larger proportions of abiraterone acetate- versus enzalutamide-treated patients received anti-androgen therapies $(57.7 \%$ vs. $48.9 \%$, respectively, including bicalutamide, nilutamide, and flutamide) and corticosteroids ( $45.6 \%$ vs. $20.0 \%$, respectively) during the baseline period. Conversely, a higher 
proportion of enzalutamide- versus abiraterone acetate-treated patients received radiation therapy $(1.4 \%$ vs. $0.4 \%$, respectively). The use of luteinizing hormone-releasing hormone agonists/antagonists and surgical castration among enzalutamide- or abiraterone acetate-treated patients was similar.

\section{Treatment Duration}

Patients treated with enzalutamide remained on treatment longer compared with patients treated with abiraterone acetate (log-rank $P=0.008$; median time to discontinuation, 10.7 vs. 8.8 months, respectively) (Fig. 2). Within 1 year of initiation, $55.7 \%$ of enzalutamide-treated patients and $60.8 \%$ of abiraterone acetate-treated patients had discontinued treatment. Similar results were observed in the subgroups of patients with diabetes, cardiovascular disease, or corticosteroid-sensitive comorbidities, with treatment duration being significantly longer among enzalutamide-treated patients (see Figs. S1-3 in the Electronic Supplementary Material). These results were also consistent in a sensitivity analysis among patients initiating their index therapy after the approval of the chemotherapy-naïve indication for enzalutamide (September 10, 2014) (Fig. 3). In this subset, treatment duration was significantly longer among enzalutamide- versus abiraterone acetate-treated patients (log-rank $P=0.016$; median time to discontinuation, 11.6 vs. 9.3 months, respectively). Furthermore, sensitivity analyses of different definitions of treatment discontinuation (i.e., gaps of $\geq 30$ or 60 days between the end of the supply of one fill for the index treatment and the date of the next fill) were consistent with the main analysis [median times to discontinuation for enzalutamide vs. abiraterone acetate (30-day gap) 8.3 vs. 8.0 months, $\log$-rank $P=0.051$; (60-day gap) 11.6 vs. 10.2 months, log-rank $P=0.008]$.

\section{HRU and Costs}

\section{All-Cause HRU and Costs}

Enzalutamide-treated patients had significantly fewer monthly all-cause inpatient admissions [adjusted IRR (95\% CI), $0.87(0.76,0.99)]$ and monthly outpatient visits $[0.94(0.90,0.98)]$ during the study period compared with abiraterone acetate-treated patients as well as fewer days of hospitalization per month 0.84 (0.70, 1.02)] (Table 2). Furthermore, within 3 months of treatment initiation, enzalutamide-treated patients were $25 \%$ less likely to have any allcause inpatient admission compared with abiraterone acetate-treated patients [adjusted OR (95\% CI), 0.75 (0.57, 0.97)] (Table 2).

With respect to healthcare costs, enzalutamide-treated patients had lower monthly costs for all-cause emergency department visits than abiraterone acetate-treated patients after adjusting for baseline covariates (adjusted difference, $-\$ 55$ per month; $P=0.018$ ) (Table 3). Conversely, the monthly all-cause pharmacy costs were higher among enzalutamide-treated patients (adjusted difference, $\$ 545$ per month; $P<0.001)$. Similar all-cause HRU and cost results were observed in the subgroup analyses among patients with diabetes, cardiovascular disease, or corticosteroid-sensitive comorbidities [see Tables S1-3 (HRU) and Tables S4-6 (costs) in the Electronic Supplementary Material].

\section{Prostate Cancer-Related HRU and Costs}

Analysis of the prostate cancer-related HRU showed enzalutamide-treated patients had significantly fewer outpatient visits during the study period [adjusted IRR (95\% CI), 0.92 (0.87, 0.96)] as well as numerically fewer inpatient visits $[0.86(0.74,1.01)]$ compared with abiraterone acetate-treated patients. Similar results were observed in the subgroup analyses among patients with diabetes, cardiovascular disease, or corticosteroid-sensitive comorbidities (see Tables S1-3 in the Electronic Supplementary Material). Furthermore, within 3 months of treatment initiation, enzalutamide-treated patients were $28 \%$ less likely to have any prostate cancer-related emergency department visits [adjusted OR (95\% CI), $0.72(0.53,0.98)]$ and $24 \%$ less likely to have inpatient admissions $[0.76(0.57,1.02)]$ than abiraterone acetatetreated patients. In terms of concomitant medication, larger proportions of abiraterone acetate- versus enzalutamide-treated patients 


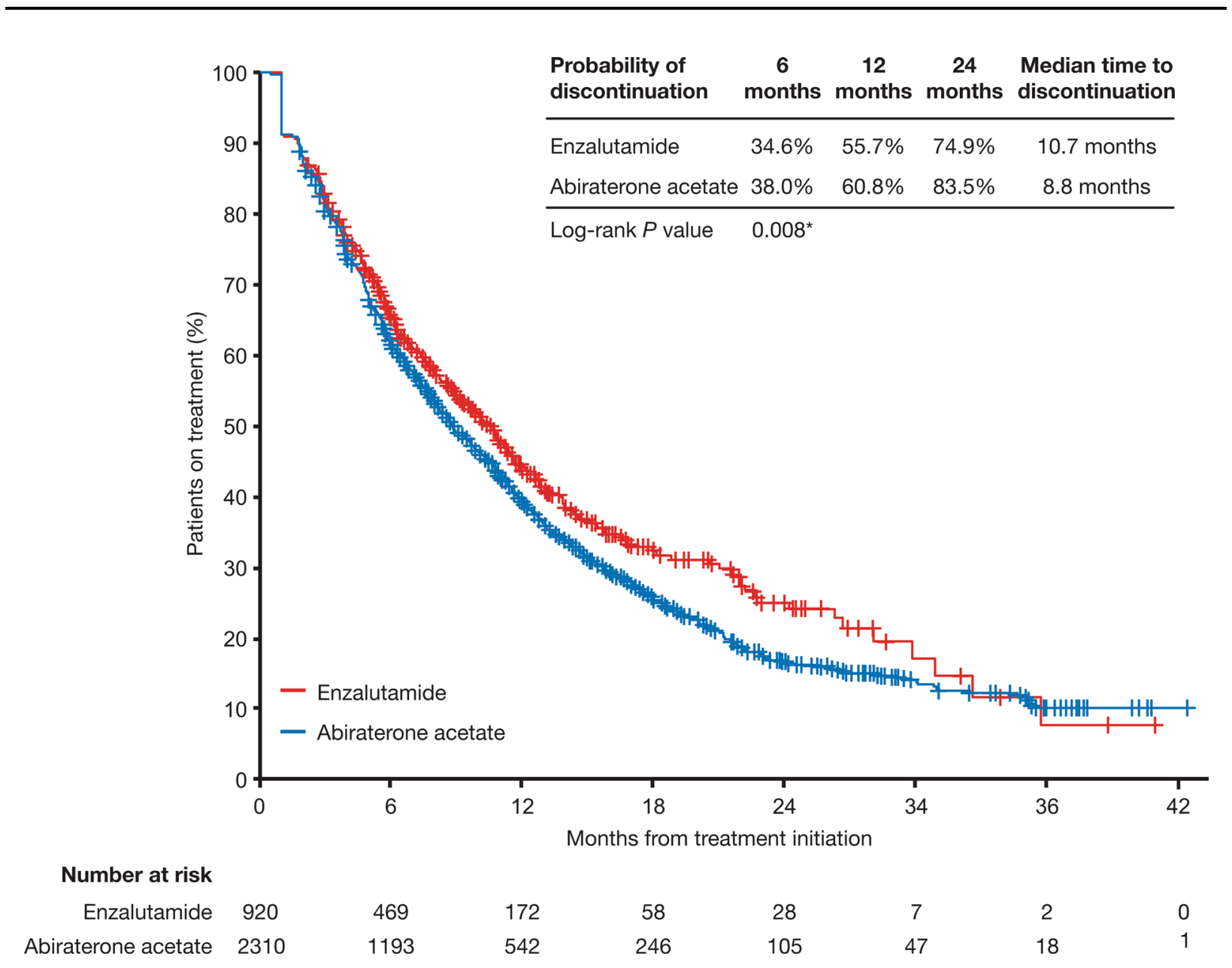

Fig. 2 Treatment duration among patients receiving enzalutamide versus abiraterone acetate. ${ }^{*} P<0.05$

received corticosteroids during the study period (89.2\% vs. $28.2 \%$, respectively), consistent with the abiraterone acetate label, which requires concurrent use with prednisone [27].

With respect to prostate cancer-related costs, enzalutamide-treated patients had significantly lower monthly economic burden due to inpatient admissions and emergency department visits compared with abiraterone acetate-treated patients after adjusting for baseline covariates. The adjusted differences (enzalutamide - abiraterone acetate) were $-\$ 122(P=0.024)$ for inpatient admissions and $-\$ 28(P=0.009)$ for emergency department visits. Also, enzalutamide-treated patients had significantly higher monthly pharmacy acquisition costs than abiraterone acetate-treated patients (adjusted differences, $\$ 485$ for total pharmacy cost and $\$ 834$ for costs of the index drug; both $P<0.001$ ).
Similar results were observed in the subgroup analyses among patients with diabetes, cardiovascular disease, or corticosteroid-sensitive comorbidities (see Tables S4-6 in the Electronic Supplementary Material).

\section{DISCUSSION}

This study provides a unique and comprehensive real-world comparison of enzalutamide and abiraterone acetate across a variety of outcomes, including treatment duration, HRU, and costs among chemotherapy-naïve patients with mCRPC. At baseline, enzalutamide-treated patients were 1 year older, on average, than abiraterone acetate-treated patients and had higher rates of certain corticosteroid-sensitive comorbidities, such as hypertension and 


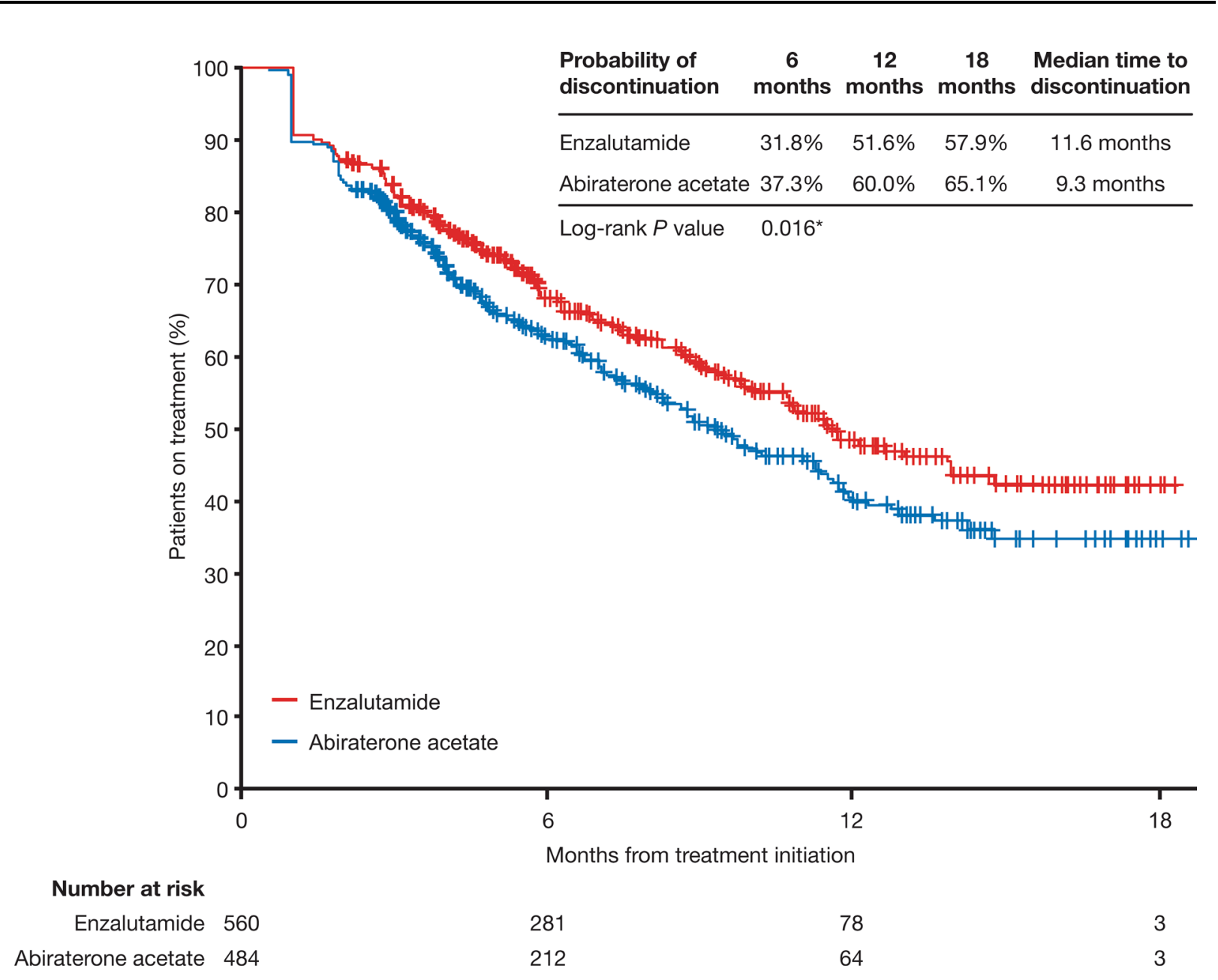

Fig. 3 Treatment duration among patients receiving enzalutamide versus abiraterone acetate (after chemotherapy-naïve indication approval for enzalutamide in September 2014). ${ }^{*} P<0.05$

diabetes. Enzalutamide-treated patients remained on treatment longer, discontinued less frequently, and had fewer all-cause and prostate cancer-related inpatient admissions and outpatient visits than patients treated with abiraterone acetate after adjusting for baseline characteristics. Despite the higher drug-acquisition cost of enzalutamide for payers, enzalutamide-treated patients had lower medical costs than those treated with abiraterone acetate, which substantially offset the calculated incremental specialty pharmacy costs of these patients. The economic results of our analyses of subgroups with specific comorbidities were consistent with the results in the overall patient cohort, supporting our main findings.
This study observed differences in the treatment durations of enzalutamide- versus abiraterone acetate-treated patients with mCRPC in the real world. Enzalutamide-treated patients remained on treatment longer than those initiating abiraterone acetate, with median treatment durations of 10.7 versus 8.8 months, respectively. In the clinical trial setting, the treatment duration of enzalutamide (PREVAIL trial, 18.2 months) [12] was also longer than that of abiraterone acetate (COU-AA-302 trial, 13.8 months) [13]. The treatment durations in the clinical trial setting were longer compared with those reported in the current real-world study of enzalutamide and abiraterone acetate, which may be explained by several factors, including discrepancies in definitions of 


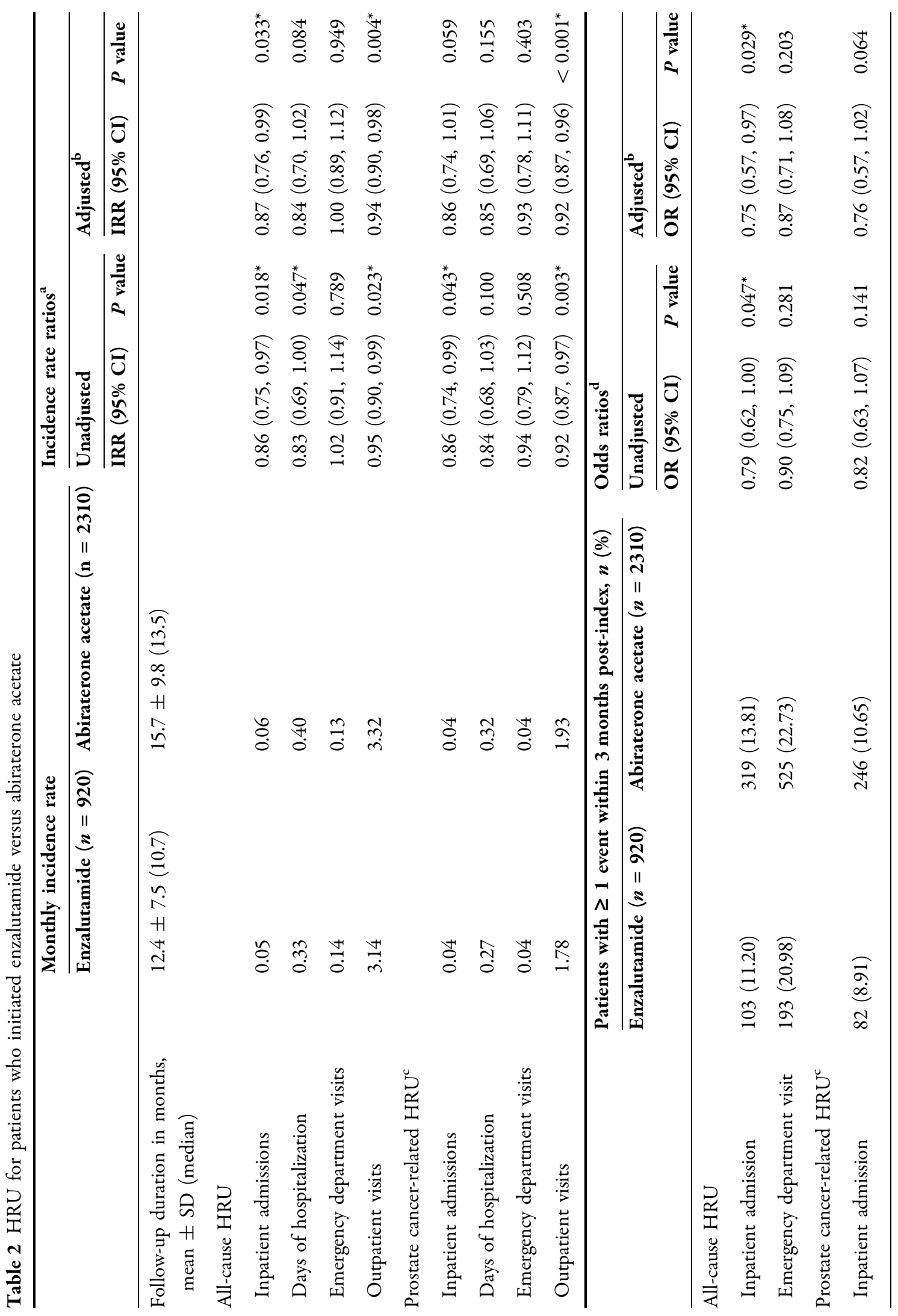




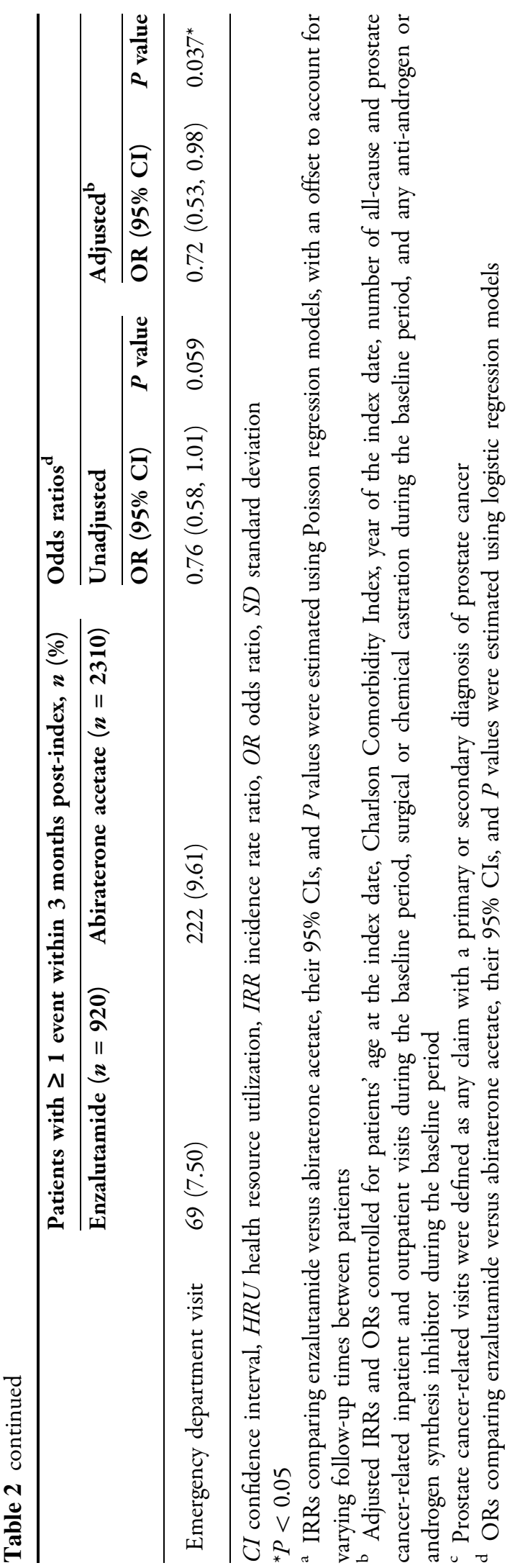

discontinuation across clinical trial and realworld settings and variations in patient populations and disease management. In general, participants in controlled clinical trials tend to be younger and have better performance status compared with patients treated in real-world clinical practice. Moreover, patients are more closely monitored in a clinical trial setting; therefore, treatment duration is generally longer than in real-world clinical practice.

Two recent administrative claims studies found conflicting results with the current study, reporting that treatment duration with abiraterone acetate was higher than with enzalutamide among overall populations of patients with prostate cancer or mCRPC [7, 17]. Pilon et al. [7] reported that the duration of overall prostate cancer treatment was significantly longer among patients who initiated abiraterone acetate compared with those who initiated enzalutamide, and a second study by Behl et al. [17] reported that patients who initiated abiraterone acetate had higher medication adherence than those who initiated enzalutamide. However, Pilon et al. and Behl et al. did not differentiate between chemotherapy-naïve and post-chemotherapy patients - two patient groups with distinct disease characteristics and severity. The chemotherapy-naïve mCRPC patient population is of particular importance, and a large retrospective study of treatment sequences for mCRPC patients from 2012 to 2014 indicated that the majority of patients using enzalutamide and abiraterone acetate were chemotherapy-naïve [16]. The current study also included an additional year of data (2012-2015) compared with Pilon et al. and Behl et al. (both 2012-2014), an important difference because the indication of enzalutamide for the treatment of chemotherapy-naïve mCRPC patients was approved in late 2014.

The restricted patient population (chemotherapy-naïve patients) and more recent data in the current analysis provide a unique contemporary view of real-world use of enzalutamide and abiraterone acetate. Because treatment duration may be considered a surrogate of treatment effectiveness in oncology, the longer observed duration of treatment among enzalutamide-treated patients may indicate superior 
Table 3 Healthcare costs (2017 US dollars) for patients who initiated enzalutamide versus abiraterone acetate

\begin{tabular}{|c|c|c|c|c|c|c|}
\hline & \multirow{2}{*}{\multicolumn{2}{|c|}{ Monthly cost, mean $\pm S D^{a}$}} & \multicolumn{4}{|c|}{$\begin{array}{l}\text { Difference in monthly cost } \\
\text { (enzalutamide }- \text { abiraterone acetate) }\end{array}$} \\
\hline & & & \multicolumn{2}{|l|}{ Unadjusted } & \multicolumn{2}{|l|}{ Adjusted $^{c}$} \\
\hline & $\begin{array}{l}\text { Enzalutamide } \\
(n=847)\end{array}$ & $\begin{array}{l}\text { Abiraterone acetate } \\
(n=2018)\end{array}$ & Difference & $P$ value & Difference & $P$ value \\
\hline $\begin{array}{l}\text { Total healthcare cost (all } \\
\text { causes) }\end{array}$ & $14,934 \pm 12,391$ & $14,691 \pm 16,094$ & 243 & 0.529 & 218 & 0.574 \\
\hline Medical service cost & $7353 \pm 12,116$ & $8211 \pm 16,152$ & -858 & $0.014^{*}$ & -90 & 0.801 \\
\hline Inpatient admissions & $1777 \pm 4487$ & $2206 \pm 5804$ & -429 & $0.008^{*}$ & -270 & 0.117 \\
\hline $\begin{array}{l}\text { Emergency department } \\
\text { visits }\end{array}$ & $285 \pm 982$ & $317 \pm 1270$ & -32 & 0.176 & -55 & $0.018^{*}$ \\
\hline Outpatient visits & $5291 \pm 10,297$ & $5689 \pm 14,002$ & -398 & 0.112 & 201 & 0.406 \\
\hline Pharmacy cost & $7581 \pm 3377$ & $6479 \pm 2929$ & 1102 & $<0.001^{*}$ & 545 & $<0.001^{*}$ \\
\hline $\begin{array}{l}\text { Total healthcare cost (prostate } \\
\text { cancer-related) }{ }^{\mathrm{d}}\end{array}$ & $11,598 \pm 7974$ & $10,975 \pm 12,051$ & 623 & $0.025^{*}$ & 458 & 0.093 \\
\hline Medical service cost & $4404 \pm 7646$ & $4835 \pm 12,060$ & -431 & 0.056 & 58 & 0.785 \\
\hline Inpatient admissions & $461 \pm 1674$ & $559 \pm 1809$ & -98 & 0.083 & -122 & $0.024^{*}$ \\
\hline $\begin{array}{l}\text { Emergency department } \\
\text { visits }\end{array}$ & $84 \pm 648$ & $111 \pm 731$ & -27 & 0.050 & -28 & $0.009^{*}$ \\
\hline Outpatient visits & $3858 \pm 7330$ & $4165 \pm 11,677$ & -307 & 0.126 & 244 & 0.197 \\
\hline Pharmacy cost ${ }^{\mathrm{e}}$ & $7194 \pm 3154$ & $6141 \pm 2665$ & 1053 & $<0.001^{*}$ & 485 & $<0.001^{*}$ \\
\hline Index drug & $6712 \pm 3304$ & $5170 \pm 2860$ & 1542 & $<0.001^{*}$ & 834 & $<0.001^{*}$ \\
\hline
\end{tabular}

$S D$ standard deviation

${ }^{*} P<0.05$

a This analysis was restricted to patients who were not on capitated insurance plans

b Cost differences and $P$ values were estimated using generalized linear models with a Tweedie distribution. Outcomes were standardized as monthly costs to account for varying follow-up times between patients

c Adjusted differences controlled for patient age at the index date, Charlson Comorbidity Index, year of index date, number of all-cause and prostate cancer-related in- and outpatient visits during the baseline period, surgical or chemical castration during the baseline period, and any anti-androgen or androgen synthesis inhibitor during the baseline period

d Prostate cancer-related visits were defined as any claim with a primary or secondary diagnosis of prostate cancer

e Prostate cancer-related pharmacy costs were defined as any claim for a prostate cancer prescription treatment

effectiveness for these patients in the real world $[28,29]$. Two independent meta-analyses of clinical trials have also been conducted on the comparative efficacy of enzalutamide and abiraterone acetate and concurred regarding the evidence that enzalutamide is superior in terms of radiographic progression-free survival and prostate-specific antigen response rate [30, 31].

This is also the first study to assess total HRU and costs of chemotherapy-naïve enzalutamideand abiraterone acetate-treated patients with mCRPC, adding a comprehensive comparison to the current knowledge base. A previous study 
by Pilon et al. used clinical trial data to compare the drug cost per median overall survival month of enzalutamide and abiraterone acetate in chemotherapy-naïve patients with mCRPC and found that enzalutamide-treated patients had higher drug costs per median overall survival month [15]. Another administrative claims study by Ellis et al. found that abiraterone acetate was associated with lower monthly pharmacy costs than enzalutamide [16]. However, neither study assessed costs beyond drug acquisition. The current study observed that the monthly pharmacy costs were higher among enzalutamide- versus abiraterone acetate-treated patients; however, the costs related to inpatient and emergency department visits were generally lower for enzalutamide-treated patients versus abiraterone acetate-treated patients. As a result, the total healthcare costs of abiraterone acetate- and enzalutamide-treated patients did not significantly differ.

Enzalutamide-treated patients had less frequent inpatient and emergency department visits compared with abiraterone acetate-treated patients. The higher rate of hospitalization observed for abiraterone acetate-treated patients could be due to several reasons. First, the current study found that higher proportions of abiraterone acetate- versus enzalutamide-treated patients had baseline corticosteroid use ( $45.6 \%$ vs. $20.0 \%$, respectively) and concomitant corticosteroid use during the study period ( $89.2 \%$ vs. $28.2 \%$, respectively). These findings are consistent with the abiraterone acetate label, which requires concurrent use with prednisone [27]. Prednisone is a required glucocorticoid replacement therapy when co-administered with abiraterone acetate, but it is associated with risks such as bone loss, immunosuppression, hyperglycemia, mood and cognitive alterations, and myopathy $[22,23,32]$. Thus, the potential side effects as well as the benefits of the prednisone regimen should be considered in conjunction. Second, in the clinical trial setting, abiraterone acetate was associated with higher rates of more serious adverse events compared with enzalutamide, including fluid retention, hypertension, and hypokalemia [13]. If the rates of more serious adverse events are also higher among abiraterone acetate-treated patients in the real world, this could explain the more frequent hospitalizations and emergency department visits by abiraterone acetate-treated patients observed in this study. The current study explored the potential reasons for HRU using the data; however, the data did not provide sufficient detail to provide the reason why costs differed between cohorts. For both cohorts, "subsequent hospital care" and "emergency department visit" were identified as the most frequent reasons for inpatient admissions and emergency department visits, respectively. The exact reasons behind the observed higher hospitalization burden on abiraterone acetate-treated patients warrant further study.

\section{Limitations}

The results of this study should be interpreted in light of several limitations. First, as with all analyses based on administrative claims data, a diagnosis code is not an attestation that the patient has the diagnosis, because the code may represent a rule-out diagnosis or may be recorded incorrectly. In the current study, at least two diagnosis codes of prostate cancer were required to mitigate the possibility of coding errors or diagnosis of exclusion. Second, the claims database does not capture important information, including medical services obtained outside of a patient's health plan (e.g., over-the-counter treatments), clinical information (e.g., reasons for treatment discontinuation), or laboratory results (e.g., prostatespecific antigen). Although the current study included both commercial and Medicare supplemental claims data to cover all age ranges, the medical services covered entirely by Medicare would not be captured in the MarketScan ${ }^{\circledR}$ data (e.g., skilled nursing facility services during the initial period after inpatient discharge). However, the proportions of patients with Medicare supplemental coverage were similar between the two cohorts $(78.8 \%$ of enzalutamide- and $75.0 \%$ of abiraterone acetate-treated patients at the index date); thus, unobserved medical services are not expected to bias the study's results. Third, there is a possibility of 
confounding due to differences in baseline characteristics. For example, more abiraterone acetate-treated patients received prior anti-androgen therapy, which indicates that these patients may have been more heavily pre-treated. To the extent possible, this study controlled for observed cohort differences at baseline, including proxies of disease severity, using multivariable regression modeling. For the treatment duration analysis, future adjusted analysis is warranted to adjust for baseline covariates. Fourth, this study used an intentionto-treat design in the comparative analyses of HRU and cost, and the cohorts were defined based on the treatment received at the index date. Future studies may consider additional methods to account for time-varying exposure and effects of the post-index treatment experience.

\section{CONCLUSIONS}

This is the first study to assess total HRU and costs of chemotherapy-naïve enzalutamide- and abiraterone acetate-treated patients with mCRPC, adding a comprehensive comparison to the current knowledge base. The results of this study suggest that differences exist between enzalutamide and abiraterone acetate in terms of the real-world treatment duration and associated HRU and costs among chemotherapynaïve patients with mCRPC. Chemotherapynaïve patients initiating enzalutamide stayed on treatment longer, incurred fewer in- and outpatient visits, and had lower prostate cancerrelated inpatient and emergency department costs. These results were largely consistent in subgroup analyses among patients with diabetes, cardiovascular disease, or corticosteroidsensitive comorbidities. Despite the higher pharmacy cost differential between enzalutamide and abiraterone acetate, the lower medical costs with enzalutamide offset the total cost burden to the healthcare system. The lower HRU burden might also reflect the superior realworld effectiveness of enzalutamide and needs to be confirmed in future studies.

\section{ACKNOWLEDGEMENTS}

Funding. Sponsorship for this study, all article processing charges, and the open access fee were funded by Astellas Pharma Inc., Northbrook, IL, USA, and Medivation LLC, a Pfizer Company, San Francisco, CA, USA, the co-developers of enzalutamide. The sponsors were involved in all aspects of the research and manuscript development. All authors had full access to all of the data in this study and take complete responsibility for the integrity of the data and accuracy of the data analysis.

Medical Writing, Editorial, and Other Assistance. The authors acknowledge Iryna Bocharova, Emily Gao, and Ellie Fuqua from Analysis Group, Inc., for significant contributions toward analytical support. Medical writing assistance was provided by Shelley Batts, PhD, an employee of Analysis Group, Inc. Editorial support was provided by Stephanie Rippon and Lauren Smith from Complete HealthVizion. Medical writing and editorial assistance were funded by the study sponsors.

Authorship. All named authors meet the International Committee of Medical Journal Editors (ICMJE) criteria for authorship for this article, take responsibility for the integrity of the work as a whole, and have given their approval for this version to be published. All authors were involved in all stages of the research, manuscript preparation, and the decision to submit.

Prior Presentations. A synopsis of the current research was presented in poster format at the ASCO Genitourinary Cancers Symposium, which took place in San Francisco, CA, USA, on February 8-10, 2018.

Disclosures. Neil M. Schultz is an employee of Astellas Pharma Inc. and owns stock in Gilead Sciences and Shire. Samuel Wilson is an employee of Astellas Pharma Inc. Scott C. Flanders was an employee of Astellas Pharma Inc. when the research was conducted and is now affiliated with Dendreon Pharmaceuticals 
LLC and owns stock in Johnson \& Johnson and AbbVie. Bruce A. Brown was an employee of Astellas Pharma Inc. when the research was conducted and is now affiliated with Dendreon Pharmaceuticals LLC. Yan Song is an employee of Analysis Group, Inc., which has received consultancy fees from Astellas Pharma Inc. Hongbo Yang is an employee of Analysis Group, Inc., which has received consultancy fees from Astellas Pharma Inc. Stanislav Lechpammer is an employee of Pfizer Inc. Vahan Kassabian is a speaker for Astellas/Pfizer, Janssen, Dendreon, Amgen, and Bayer; a consultant for Dendreon, UroGPO, Bayer, Tolmar, Janssen, Astellas/Pfizer; an employee of Georgia Urology; and owns UroGPO stock.

Compliance with Ethics Guidelines. This was a retrospective analysis of anonymized data; no institutional board review was required.

Data Availability. Access to anonymized individual participant-level data will not be provided for this study as the data meet one or more of the exceptions described on http:// www.clinicalstudydatarequest.com under "Sponsor Specific Details for Astellas."

Open Access. This article is distributed under the terms of the Creative Commons Attribution-NonCommercial 4.0 International License (http://creativecommons.org/licenses/ by-nc/4.0/), which permits any noncommercial use, distribution, and reproduction in any medium, provided you give appropriate credit to the original author(s) and the source, provide a link to the Creative Commons license, and indicate if changes were made.

\section{REFERENCES}

1. Ferlay J, Soerjomataram I, Ervik M, et al. GLOBOCAN 2012 v1.0, Cancer incidence and mortality worldwide: IARC CancerBase no. 11. 2014. http:// globocan.iarc.fr. Accessed 22 Mar 2018.

2. Siegel RL, Miller KD, Jemal A. Cancer statistics, 2016. CA Cancer J Clin. 2016;66(1):7-30.
3. Sonpavde G, Pond GR, Armstrong AJ, et al. Radiographic progression by Prostate Cancer Working Group (PCWG)-2 criteria as an intermediate endpoint for drug development in metastatic castration-resistant prostate cancer. BJU Int. 2014;114(6b):E25-31.

4. Kirby M, Hirst C, Crawford ED. Characterising the castration-resistant prostate cancer population: a systematic review. Int J Clin Pract. 2011;65(11):1180-92.

5. National Cancer Institute. Prostate cancer treatment-health professional version $\left(\mathrm{PDQ}^{\circledR}\right)$. 2018. http://www.cancer.gov/types/prostate/hp/prostatetreatment-pdq. Accessed 7 Jul 2018.

6. Armstrong AJ, George DJ. New drug development in metastatic prostate cancer. Urol Oncol. 2008;26(4):430-7.

7. Pilon D, Behl AS, Ellis LA, Emond B, Lefebvre P, Dawson NA. Duration of treatment in prostate cancer patients treated with abiraterone acetate or enzalutamide. J Manag Care Spec Pharm. 2017;23(2):225-35.

8. National Comprehensive Cancer Network. NCCN clinical practice guidelines in oncology (NCCN guidelines $^{\circledR}$ ). Prostate cancer. 2016. http://www.trikobe.org/nccn/guideline/urological/english/prostate. pdf. Accessed 11 Apr 2017.

9. Roviello G, Sigala S, Sandhu S, et al. Role of the novel generation of androgen receptor pathway targeted agents in the management of castrationresistant prostate cancer: a literature based metaanalysis of randomized trials. Eur J Cancer. 2016;61:111-21.

10. Ning YM, Brave M, Maher VE, et al. U.S. Food and Drug Administration Approval Summary: enzalutamide for the treatment of patients with chemotherapy-naive metastatic castration-resistant prostate cancer. Oncologist. 2015;20(8):960-6.

11. Schalken J, Fitzpatrick JM. Enzalutamide: targeting the androgen signalling pathway in metastatic castration-resistant prostate cancer. BJU Int. 2016;117(2):215-25.

12. Beer TM, Armstrong AJ, Rathkopf D, et al. Enzalutamide in men with chemotherapy-naive metastatic castration-resistant prostate cancer: extended analysis of the phase 3 PREVAIL study. Eur Urol. 2017;71(2):151-4.

13. Ryan CJ, Smith MR, Fizazi K, et al. Abiraterone acetate plus prednisone versus placebo plus prednisone in chemotherapy-naive men with metastatic castration-resistant prostate cancer (COU-AA-302): final overall survival analysis of a randomised, 
double-blind, placebo-controlled phase 3 study. Lancet Oncol. 2015;16(2):152-60.

14. National Comprehensive Cancer Network. NCCN clinical practice guidelines in oncology (NCCN Guidelines $\left.{ }^{\circledR}\right)$. Prostate cancer. Version 3. 2018. https://www.nccn.org/professionals/physician_gls/ pdf/prostate.pdf. Accessed 7 Jul 2018.

15. Pilon D, Queener M, Lefebvre P, Ellis LA. Cost per median overall survival month associated with abiraterone acetate and enzalutamide for treatment of patients with metastatic castration-resistant prostate cancer. J Med Econ. 2016;19(8):777-84.

16. Ellis LA, Lafeuille MH, Gozalo L, Pilon D, Lefebvre $\mathrm{P}$, McKenzie S. Treatment sequences and pharmacy costs of 2 new therapies for metastatic castrationresistant prostate cancer. Am Health Drug Benefits. 2015;8(4):185-95.

17. Behl AS, Ellis LA, Pilon D, Xiao Y, Lefebvre P. Medication adherence, treatment patterns, and dose reduction in patients with metastatic castration-resistant prostate cancer receiving abiraterone acetate or enzalutamide. Am Health Drug Benefits. 2017;10(6):296-303.

18. Scher HI, Beer TM, Higano CS, et al. Antitumour activity of MDV3100 in castration-resistant prostate cancer: a phase 1-2 study. Lancet. 2010;375(9724):1437-46.

19. Attard G, Reid AH, A'Hern R, et al. Selective inhibition of CYP17 with abiraterone acetate is highly active in the treatment of castration-resistant prostate cancer. J Clin Oncol. 2009;27(23):3742-8.

20. Beer TM, Armstrong AJ, Rathkopf DE, et al. Enzalutamide in metastatic prostate cancer before chemotherapy. N Engl J Med. 2014;371(5):424-33.

21. Ryan CJ, Smith MR, de Bono JS, et al. Abiraterone in metastatic prostate cancer without previous chemotherapy. N Engl J Med. 2013;368(2):138-48.

22. Bui CN, Wang L, Baser O. Resource utilization and use of life-extending therapies and corticosteroids in prostate cancer patients with corticosteroid-sensitive comorbidities. Curr Med Res Opin. 2014;30(11):2355-64.
23. Quan H, Sundararajan V, Halfon P, et al. Coding algorithms for defining comorbidities in ICD-9-CM and ICD-10 administrative data. Med Care. 2005;43(11):1130-9.

24. Kaas R. Compound Poisson distribution and GLM's-Tweedie's distribution. In: Vanmaele M, De Schepper A, Dhaele J, Reynaerts H, Schoutens W, Van Goethem P, editors. Proceedings of the 3rd actuarial and financial mathematics day. Wetteren: Universa Press; 2005. p. 3-12.

25. Klinker F. Generalized linear mixed models for ratemaking: a means of introducing credibility into a generalized linear model setting. CAS E-Forum. 2011;2(Winter):1-25.

26. Smyth GK, Jorgensen B. Fitting Tweedie's Compound Poison Model to insurance claims data: dispersion modelling. ASTIN Bull. 2002;32(1):143-57.

27. Food and Drug Administration. Zytiga [package insert]. 2016. http://www.accessdata.fda.gov/ drugsatfda_docs/label/2015/202379s016lbl.pdf. Accessed 22 Mar 2018.

28. Jensen GA, Li Y. Long-run health effects of costrelated non-adherence to prescribed medications among adults in late midlife. JPHSR. 2012;3(2):85-93.

29. McCowan C, Shearer J, Donnan PT, et al. Cohort study examining tamoxifen adherence and its relationship to mortality in women with breast cancer. Br J Cancer. 2008;99(11):1763-8.

30. Chopra A, Georgieva M, Lopes G, Yeo C, Haaland B. Abiraterone or enzalutamide in advanced castration-resistant prostate cancer: an indirect comparison. Prostate. 2017;77(6):639-46.

31. Fang M, Nakazawa M, Antonarakis ES, Li C. Efficacy of abiraterone and enzalutamide in pre- and postdocetaxel castration-resistant prostate cancer: a trial-level meta-analysis. Prostate Cancer. 2017;2017:8560827.

32. Auchus RJ, Yu MK, Nguyen S, Mundle SD. Use of prednisone with abiraterone acetate in metastatic castration-resistant prostate cancer. Oncologist. 2014;19(12):1231-40. 